\title{
Compliance for uncertain inventories via probabilistic/fuzzy comparison of alternatives
}

\author{
Olgierd Hryniewicz $\cdot$ Zbigniew Nahorski • \\ Jörg Verstraete • Joanna Horabik • Matthias Jonas
}

Received: 22 December 2012 / Accepted: 6 December 2013 / Published online: 15 February 2014

C) The Author(s) 2014. This article is published with open access at Springerlink.com

\begin{abstract}
A direct comparison among highly uncertain inventories of emissions is inadequate and may lead to paradoxes. This issue is of particular importance in the case of greenhouse gases. This paper reviews the methods for the comparison of uncertain inventories in the context of compliance checking. The problem is treated as a comparison of uncertain alternatives. It provides a categorization and ranking of the inventories which can induce compliance checking conditions. Two groups of techniques to compare uncertain estimates are considered in the paper: probabilistic and fuzzy approaches. They show certain similarities which are revealed and stressed throughout the paper. The group of methods most suitable for the compliance purpose is distinguished. They introduce new conditions for fulfilling compliance, depending on inventory uncertainty. These new conditions considerably change the present approach, where only the reported values of inventories are accounted for.
\end{abstract}

\section{Introduction}

A handful of solutions have been proposed to cope with the problem of emission commitment evaluation for uncertain inventories, see Jonas and Nilsson (2007). Numerous propositions have pointed to methodological incompetence in using the reported (point) values in clearing emission targets. For many environmental problems such as for greenhouse gases, only highly imprecise values of emission are available, see e.g. Jonas and Nilsson (2007); Jonas et al. (2010b); Lieberman et al. (2007); White et al. (2011). Apart from a high uncertainty level, uncertainty distributions are often asymmetric, as they reflect non-negative measurements of physical quantities. For an example, see the results in Ramirez et al. (2006) or Winiwarter and Rypdal (2001).

This article is part of a Special Issue on "Third International Workshop on Uncertainty in Greenhouse Gas Inventories" edited by Jean Ometto and Rostyslav Bun.

Electronic supplementary material The online version of this article (doi:10.1007/s10584-013-1031-x) contains supplementary material, which is available to authorized users.

O. Hryniewicz $\cdot$ Z. Nahorski $(\bowtie) \cdot J$. Verstraete $\cdot$ J. Horabik

Systems Research Institute, Polish Academy of Sciences, Newelska 6, 01-447 Warsaw, Poland

e-mail: Zbigniew.Nahorski@ibspan.waw.pl

M. Jonas

International Institute for Applied Systems Analysis, Schlossplatz 1, 2361 Laxenburg, Austria 
According to the IPCC Good Practice Guidelines (IPCC 1996), a report should be "consistent, comparable and transparent". Decisions on the fulfilment of obligations should be fair for all parties, which means that it should be transparent why some inventories comply with commitments while others do not. Since greenhouse gases inventories are highly uncertain, making decisions on compliance or comparison of inventories based only on the reported values (estimated size) may contradict any conclusions inferred from considering uncertainty distributions such as uncertainty range (e.g. standard deviation) and the shape of uncertainty distribution (e.g. skewness). We argue that this knowledge should be fully utilized to make decisions on compliance and to infer a comparison of emissions.

Let us consider two uncertain emission inventories, A and B of Fig. 1a-b, which will help us to illustrate the techniques discussed. For the sake of simplicity, let us assume that both involved parties have the same emission limits, also called a target, for instance, an allocated number of emission permits. The dominant values of the uncertainty distribution densities $\mu(x)$ reflect the reported inventories of both parties, which are very close. If uncertainty is ignored, party A would be considered compliant (fulfilling the limit), while party B would not. However, confidence in the inventory value of party $\mathrm{B}$ is high, while that of $\mathrm{A}$ is low, raising the question which party is more credible? Should party A be considered compliant, while party B should not? Certainly, to compare parties with different scale of emissions, the inventories have to be normalized. For example, the value $d=(x-K) / x$, with $K$ denoting the party's emission limit, may be a suitable normalization; then the normalized limit is equal to zero. Henceforth, the term inventory will always refer to a normalized inventory.

a

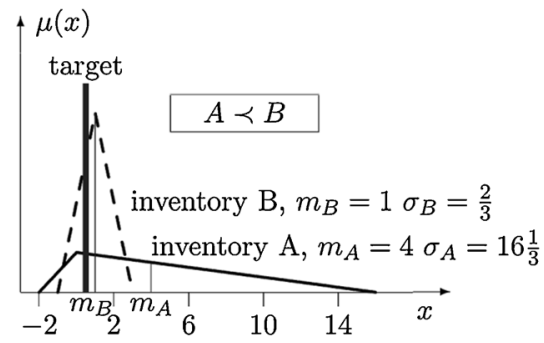

b

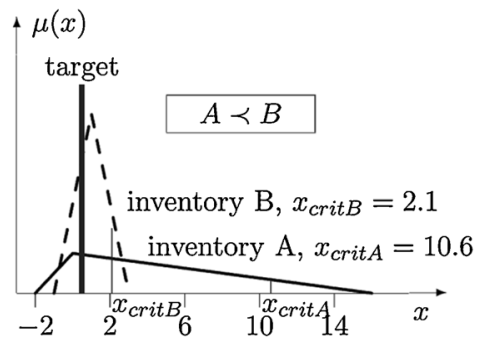

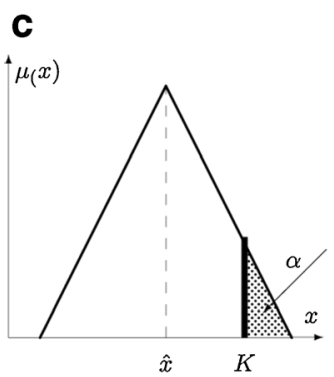

d

e
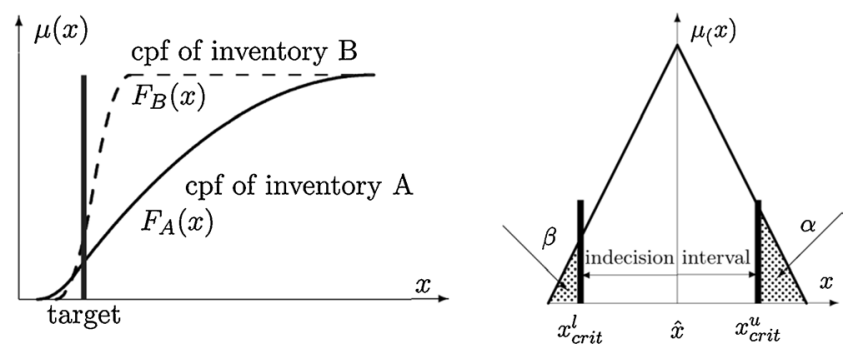

Fig. 1 Illustration for statistical approaches: a Comparison of means and variances; b Calculation of critical values; c Illustration of compliance in the undershooting approach; d Stochastic dominance criterion for comparison of inventories A and B; e The indecision interval 
In this paper we look at the problem of fulfillment as one of the comparison and ordering of (normalized) inventories. When inventories can be ordered in a transparent way, then we can point to the threshold value, below which the inventories are compliant, and above which they are not. With the present method inventories care actually ordered according to the reported values. Those which are below the limit are considered compliant, while those above are not. As mentioned before, the ordering of uncertain inventories simply according to their reported values is dubious, since their uncertainty should also be taken into account. The idea of this paper is to review the methods for comparison of uncertain values, so that uncertain inventories can be credibly ordered. This will introduce transparency into the compliance mechanism. Henceforth, a higher ranked inventory $A$ is considered better in respect to (i.r.t.) the target than inventory $B$, and is denoted by $B \prec A$.

Verification of emission reduction in a single country may also face the problem of uncertainty. Let us consider the case of greenhouse gases, when a reduction of emissions at the end of the commitment period is required. This is expressed as a specified rate $\rho$ of the real emissions in the previous (basic) year. Since the real emissions are not known, only an uncertain inventory in the compliance year, $x_{c}$, can be compared with a reduced uncertain inventory in the basic year, $\rho x_{b}$. From this, it has to be decided whether the former emissions are lower than the latter. In other words, these two inventories should be ranked in as convincing a manner as possible. This also motivated our search for adequate methods to compare uncertain values. But another view is also possible in this case. Consider a variable defined as $d=\frac{x_{c}}{x_{b}}-\rho$ or the so called trend uncertainty $d=\frac{x_{b}-x_{c}}{x_{b}}-(1-\rho)$. These are normalized variables, which can be used for comparison among countries with different scales of emissions, and even with different reduction rates $\rho$. For them, the limit for comparison equals zero. Consequently, countries can be ordered appropriately according to their values of $d$ and accounting for uncertainty. With this ordering, compliance conditions can be formulated. Note that for the Monte Carlo simulation, which is presently the basic tool for assessment of uncertainty distributions, there is no great difference as to whether a distribution of variable $d$ instead of variable $x$ is to be generated.

Nevertheless, it should be stressed that ranking is only supplementary to the compliance checking rule that is adopted. It can help to justify why some inventories are considered compliant, while others are not. Ranking of inventories may facilitate avoiding paradoxical situations, when decisions on compliance or noncompliance are at variance with common sense.

Throughout the paper it is assumed that the distribution of inventory uncertainty is available. This would be the ideal case. Unfortunately, for national GHG inventories it is largely impossible to estimate distributions in the statistical sense, since inventories cannot be repeated in great numbers with different values of unsure parameters. The distributions can be, however, assessed by performing Monte Carlo calculations, which provide good insight to the distribution of national inventories. Some countries (e.g. Austria, the Netherlands) have undertaken this effort (Winiwarter and Rypdal 2001; Ramirez et al. 2006). Others report either uncertainty intervals or simply standard deviations. Although the probability-rooted methods presented in Section 2 mostly require knowledge of probability distribution, in the fuzzy-set-rooted methods, discussed in Section 3, the distribution of uncertainty may be shaped more flexibly, including interval information or, for instance, the use of expert knowledge. The assessment of uncertainty distribution and the accuracy of its estimation is a problem in itself. It requires a separate discussion, which, however, is beyond the scope of the present paper. 


\section{Probabilistic approaches}

\subsection{Introductory remarks}

Although the inventories do not fully comply with randomness assumptions, treating an inventory as a random value with probabilistic distribution seems to be self-imposing.

The comparison of uncertain random values has been already considered in various fields. The problem of selection from high-risk projects has had a long history in areas such as finance, R\&D projects, or IT projects (Graves and Ringuest 2009). Several methods have been proposed to compare such projects. The methods can be further divided into groups. All the methods presented below are adapted to the problem of emission inventories. Most of them require knowledge of the inventory probability distribution.

\subsection{Statistical moments}

Mean value and variance The most elementary technique is based on the mean value and variance (MV). Obviously, the smaller the mean value and variance, the better the inventory. This approach is illustrated in Fig. 1a. Although the reported value of inventory $\mathrm{A}$ is smaller than that of $\mathrm{B}$, its mean value is greater than the mean value of $\mathrm{B}$. The same is true for the standard deviations. Even this simple criterion shows that an inventory of party B should be considered better i.r.t. the target than that of party A. This is contradictory to the result based on reported values, which disregard uncertainty. According to the latter approach, the compliance mechanism would be related to a comparison of mean values, and not reported values. However, a single mean value is not enough for ranking purposes.

Semivariance Comparison of inventories using two indices, mean value and standard deviation, may lead to contradictory results. A notion of the semivariance (MSV) should rather be applied, following the definition

$$
s_{S}^{2}=\int_{K}^{\infty}(x-K)^{2} \mu(x) d x,
$$

where $K$ is a chosen value and $\mu(x)$ is the distribution density function of an inventory. The smaller the value of $s_{S}^{2}$, the higher the inventory is ranked. In our case, $K$ can be conveniently chosen as a given target, and this value is used in the example of Fig. 1a, as well as in the result survey of Table S1 in the supplementary material. In the considered example it holds that $s_{S A}^{2}>$ $s_{S B}^{2}$, thus, inventory B is better i.r.t. the target than A. According to the criterion, an inventory satisfies the target if the semivariance is smaller than a preselected value.

\subsection{Critical values}

Critical probability A large group of techniques use the term critical probability (CP), a notion first introduced in 1952 (Roy 1952). It is defined as the probability of surpassing target $K$

$$
c r p=\int_{K}^{\infty} \mu(x) d x
$$


A smaller value of $c r p$ indicates a better inventory i.r.t. the target. As seen in Fig. 1b, again, an inventory of party B is evaluated as being better. Determining compliance with a limit is based on calculation of the critical probability, which should not be greater than any prescribed value.

Risk In other related methods, as the Baumol's risk measure and the value at risk (VaR), a critical value $x_{\text {crit }}$ is calculated for a settled probability $\alpha$, so that the probability that emission value will be higher than $x_{\text {crit }}$ is $\alpha$. Without going into details, an inventory is better i.r.t. the target whenever $x_{\text {crit }}$ is smaller. In the example from Fig. $1 \mathrm{~b}$, with fixed probability $\alpha=0.1$, inventory $\mathrm{B}$ is indicated as the better one.

Undershooting A technique similar in spirit has been proposed to ensure reliable compliance. It states that only a small enough $\alpha$-th part of an inventory distribution may lie above target $K$. This approach is called undershooting, see Gillenwater et al. 2007; Godal et al. 2003; Nahorski and Horabik 2010; Nahorski et al. 2003, and it is illustrated in Fig. 1c. Note, that when used for ordering inventories, the idea becomes equivalent to the $\mathrm{CP}$ technique.

\subsection{Stochastic dominance}

Stochastic dominance In the stochastic dominance technique inventory B is better i.r.t. the target than $\mathrm{A}$ if their cumulative probability functions (cpf s) satisfy $F_{A}(x) \leq F_{B}(x)$ for all $x$, and the condition is strict for at least one $x$. It is obvious that not all inventories can be decisively compared this way, see $c p f$ s of our exemplary inventories $\mathrm{A}$ and $\mathrm{B}$ depicted in Fig. 1d. Although cpf of party B is greater for most values of $x$, it is lower than $c p f$ of party A for a small range of low values. This potential lack of an unequivocal answer is a serious drawback of the method. However, some modifications have been proposed to extend its usability.

Almost stochastic dominance In the almost stochastic dominance (ASD) ${ }^{1}$ inventory B is better i.r.t. the target than $\mathrm{A}$, if the area between both $c p f \mathrm{~s}$ for $F_{B}(x)<F_{A}(x)$ is a small enough ( $\varepsilon$ times smaller, usually with $0<\varepsilon<0.5)$ part of the whole area between $p d f \mathrm{~s}, \int_{x}\left|F_{B}(x)-F_{A}(x)\right| d x$. It can be seen by inspection of Fig. 1d that this condition is satisfied in our example of Fig. 1a-b. Thus, this technique also indicates inventory B better i.r.t. the target.

A simplified comparison of inventories would confine itself to checking the values of $c p f \mathrm{~s}$ at $x=K$. This would be equivalent to a variant of critical probability approach. Thus, the analysis of fulfilment of the limit in the stochastic dominance techniques could be reduced to checking if the value of the inventory $c p f$ at the limit is sufficiently high.

\subsection{Two-sided comparison of inventories}

The approaches discussed guarantee a proper ordering when the reported value is smaller or equal to the limit $K$ (see supplementary material). To properly order the inventories for $K<\widehat{x}$, it is useful to consider the probability

$$
\beta=\int_{-\infty}^{K} \mu(x) d x .
$$

\footnotetext{
${ }^{1}$ This is the first order ASD. For the second order ASD see Graves and Ringuest (2009).
} 
The smaller the value of $\beta$ is, the more certain the inventory is likely to be noncompliant. To make significant decisions, we would like to have a small value of $\alpha$ to help decide that the inventory is compliant, and a small value of $\beta$ to help decide that it is noncompliant. Having fixed $\alpha$ and $\beta$, we can calculate the corresponding critical values $x_{c r i t}^{u}$ and $x_{c r i t}^{l}$, as illustrated in Fig. 1e. Thus, there will be an indecision interval for $K \in\left(x_{c r i t}^{l} x_{c r i t}^{u}\right)$ where there is uncertainty as to whether the inventory fulfils the limit or not. This can be considered as a generalization of the undershooting method.

The question arises what can be done when the limit falls into the indecision interval. It is actually fair to say that no decision can be taken confidently. One of the answers proposed in Jonas et al. (1999) and Gusti and Jęda (2002) was to wait until the inventory subsequently crosses an indecision boundary in the consequent years. A rough method to estimate when this may take place was also designed, called the verification time. It is based on a linear or quadratic prognosis of future emission trajectory combined for compliance with an obligatory undershooting of the indecision boundary, so that the national emission reductions and limitations become detectable.

\section{Fuzzy set approaches}

\subsection{Introduction}

Fuzzy set and possibilistic models of uncertainty can be considered as a competitive approach to the probabilistic one, described above. In the fuzzy set theory, comparison and ranking of fuzzy (or inaccurate) values is a problem to which different solutions have been proposed. Ignoring conceptual differences, there are sufficient similarities to warrant further investigation into how the possibilistic ranking methods hold up against the other methods. In the following subsections, we will list four conceptually different groups of methods that are used to compare fuzzy numbers. Some of the methods resemble those from the probabilistic approaches; others use different paradigms. The methods illustrate the fact that various approaches can be used to tackle the comparison problem.

A short introduction to the fuzzy sets and discussion of conceptual differences between the probabilistic and fuzzy set approaches can be found in the supplementary material.

\subsection{On the underlying assumptions}

Most of the fuzzy comparison and ranking methods have been developed for fuzzy sets over the domain $[0,1]$. The main reason for this is that there are some specific advantages in developing ranking methods (e.g. integrals over the domain cannot yield a result greater than 1). For the application of the methods in the comparison and ranking of different inventories, the methods could be modified to suit a different domain. This is possible for all the methods, but may complicate the formulas somewhat. To keep the formulas simple and to remain true to the original definitions, this option was disregarded. An alternative option would be to rescale the domain of the inventories to the interval $[0,1]$ to allow for a direct application of the methods. If the supports of the fuzzy number is finite, as we assume here, and in the original support $x \in[l, r]$, the new variable, spread in $[0,1]$, is defined as $z=(x-l) /(r-l)$.

The ranking methods below put forward a comparison of at least two fuzzy numbers. Some authors have chosen to rank from lowest to highest; others rank from highest to lowest. The aim of this article is to present different methods and show how difficult cases can be distinguished differently. Although these are minor details that can easily be overcome this should not detract anything from the message. 
Not all the techniques proposed for comparison of fuzzy sets are mentioned below. Some of those not mentioned can be found in a review paper by Bortolan and Degani (1985). A more recent technique can be found in Tran and Duckstein (2002).

\subsection{An analogue to moments}

Yager F1 In Yager (1981), three different ranking methods are presented. They are pure ranking methods in the sense that a number is derived for every element. This number is independent of the other elements in the set.

A weight function $g$ is introduced to add weights to the fuzzy set $A$. Basically, this allows us to specify which values are more important, based on their possibility. Common weight functions are either $g(z)=1$ (reflecting that all possible values are equally important) or $g(z)=$ $z$ (indicating that the higher the possibility of a value, the more important it is and the more it will contribute to determine the rank).

The first ranking function is defined as follows:

$$
F_{1}(A)=\frac{\int_{0}^{1} g(z) \mu_{A}(z) d z}{\int_{0}^{1} \mu_{A}(z) d z} .
$$

If the weight function $g(z)=z$ is used, then $F_{1}$ represents the mean value of the membership function, called usually the center of gravity of the fuzzy set. This is illustrated in Fig. 2a. Note that if the weight function $g(z)=1$ is used; no ranking conclusions can be drawn: $F_{1}$ would result in 1 for every fuzzy set.

When $g(z)=z$, this technique can be compared with the mean value technique in the probabilistic approach. The ranking function may be defined in a more general way, and one option could be to take $g(z)=\left[z-\left.F_{1}(A)\right|_{g(z)=1}\right]^{2}$, as analogous to the variance. An analogue of semivariance could also be defined here, which shows the similarity of this fuzzy approach technique with the probabilistic one.

\subsection{Analogues to critical values}

Nahorski et al A strict analogue to a critical value technique in the probabilistic approach has been proposed in Nahorski et al. (2003); Nahorski et al. (2007); Nahorski and Horabik (2010). To get an analogue to probability, which defines the critical value, the critical area is normalized by dividing it by the area under the membership function, as in Fig. 1c.

Adamo On the other hand, Adamo (1980) proposed to consider points fulfilling $\mu_{A}(z)=\alpha$, $0 \leq \alpha \leq 1$ and choose the highest value of $z$ as a ranking criterion. In other words, the criterion value is the rightmost value of the $\alpha$-cut of the fuzzy number $A$. The critical value now depends on the choice of $\alpha$, but in this case it has a clear fuzzy set interpretation connected with the $\alpha$-cut. This idea can be compared with the one by Nahorski et al., where the critical area has a more probabilistic origin, while that of Adamo has more the flavour of a fuzzy set, see Fig. 2d. Both techniques can be related by mathematical expressions for a fixed membership function.

These techniques can be simply used for the derivation of criterions for checking the fulfilment of the limit, analogously to those which stem from similar probabilistic approaches. 

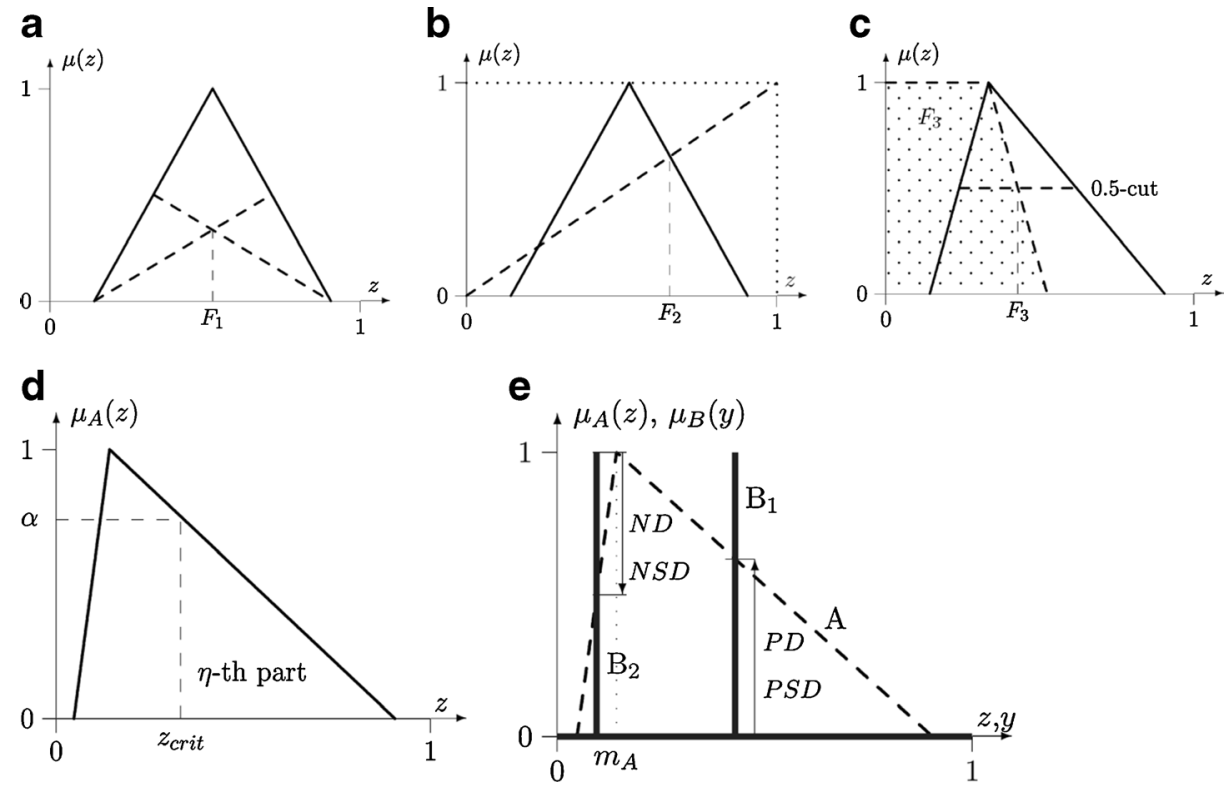

Fig. 2 Illustration for fuzzy set approaches. Ranking functions proposed by Yager: a F1 function; b F2 function; c F3 function; d Determination of the critical value $z_{\text {crit }}$ in the Nahorski et al. (calculation of the $\eta$-th part of the distribution area) and Adamo (calculation of the $\alpha$-cut) techniques; e Calculation of the indices for the crisp limits

Yager F2 The second ranking function introduced by Yager (1981) compares the given fuzzy set $A$ to the linear fuzzy set $B$, defined by $\mu_{B}(z)=z$.

The second ranking function is then defined as follows:

$$
F_{2}(A)=\max _{z \in S} \min \left(z, \mu_{A}(z)\right) .
$$

Here, $S$ represents the support of the fuzzy set $A$; in our case assumed to be the interval $[0,1]$. Graphically, this yields an intersection point between the linear fuzzy set $\left(\mu_{B}(z)=z\right)$ and the given fuzzy set $A$. This is illustrated in Fig. $2 \mathrm{~b}$.

This ranking function has a simple interpretation. The fuzzy set with the membership function $\mu_{B}(z)=z$ may be interpreted as representing a variable "high". The membership function $\min \left(z, \mu_{A}(z)\right)$ represents a variable, which is a conjunction of $A$ and $B$, i.e. the points which belong both to the variable "high" and $A$. In other words, it represents a distribution of the possibility that $A$ is "high". Its maximal point satisfies these two requirements in the "best" way.

The membership function of the variable "high" may be shaped in a different way. Jain (1976) proposed a more general set of functions $\mu_{B}(z)=\left(z / z_{\max }\right)^{k}, k>0 .{ }^{2}$ In this case, the result of a comparison of fuzzy numbers may largely depend on the choice of $k$, though no clear criteria exist for which value of $k$ should be chosen.

Apart from ranking the fuzzy numbers, the critical values could be used to check on the fulfilment of obligations, analogously to the stochastic approach. The simplest approach would be to directly compare $F_{2}$ with $K$. However, the constructions proposed here are of a subjective character and remain difficult to interpret physically, and therefore their use may be limited.

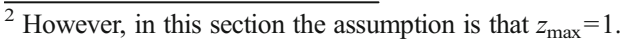


Yager F3 The third ranking function defined by Yager (1981) is more complex to explain through the use of formulae, although it is simple to interpret geometrically. It is defined as

$$
F_{3}(A)=\int_{0}^{\alpha_{\max }} m\left(A_{\alpha}\right) d \alpha .
$$

with $A_{\alpha}$ the $\alpha$-cuts of $A, \alpha_{\max }$ is the highest occurring possibility in the fuzzy set $A,{ }^{3}$ and $m$ is the middle point of the $\alpha$-cut.

The formula is relatively easy to grasp graphically: the index is the surface area to the left of the line that runs exactly along the middle of the fuzzy number. For triangular fuzzy numbers, this connects the top of the fuzzy number (i.e. where the possibility is one) with the middle of the support. This is represented by the shaded area in Fig. 2c.

This ranking index can be directly used for checking the satisfaction of the limit. For this we ought to remain aware that $F_{3}(A)$ is the mean value of the function $m\left(A_{\alpha}\right)$, in which $\alpha$ is the argument. This is because $0 \leq \alpha \leq 1$, so for the triangular membership functions $F_{3}(A)=$ $\int_{0}^{1} m\left(A_{\alpha}\right) d \alpha=m\left(A_{0.5}\right)$. Thus, in this case $F_{3}(A)$ is equal to the middle value of the 0.5 -cut of the fuzzy number $A$, see Fig. 2c. For other membership functions the integral will be equal to the middle value of some $\alpha$-cut, possibly different from 0.5. Clearly, this index is closely related with an $\alpha$-cut, where the appropriate $\alpha$ is determined by the shape of the membership function. It makes this approach slightly similar to the Adamo method, with the critical value determined in the middle of the $\alpha$-cut instead of at the right end. This interpretation encouraged us to classify this technique within the critical values group.

Examples with a comparison of the Yager ranking methods can be found in the supplementary material.

\subsection{Fuzzy dominance}

\subsubsection{Possibility and necessity measures}

In spite of its similar name, the fuzzy dominance techniques proposed to date in the literature, differ completely in spirit from the stochastic dominance ones that are presented in subsection 2.4. It is important to remember here that we use the normalized fuzzy numbers on the domain rescaled to the interval $[0,1]$. The results of this subsection may be not correct if the normalization or rescaling is not conducted beforehand.

To compare fuzzy numbers using the fuzzy dominance approach, possibility and necessity measures can be used, as introduced by Dubois and Prade (1983), see also Hryniewicz and Nahorski (2008). A normalized fuzzy set with a membership function $\mu(z)$ induces a possibility distribution $\pi(z)=\mu(z)$ on the interval $[0,1]$. For simplicity, we refer to possibility distribution as $\mu(z)$. Given a possibility distribution, the possibility measure of a subset $Z \in U=[0,1]$ is defined as

$$
\operatorname{Poss}(Z)=\sup _{z \in Z} \mu(z) .
$$

It can be interpreted as a degree of possibility that an element is located in set $Z$, see an interpretation in Fig. 3a. Let us draw attention to the fact that using a characteristic function $\chi_{Z}(z)$ of the set $Z$, the possibility measure can be equivalently defined as

$$
\operatorname{Poss}(Z)=\sup _{z \in[0,1]} \min \left\{\mu(z), \chi_{Z}(z)\right\} .
$$

\footnotetext{
${ }^{3}$ For the normalized sets, as assumed in this paper, $\alpha_{\max }=1$.
} 

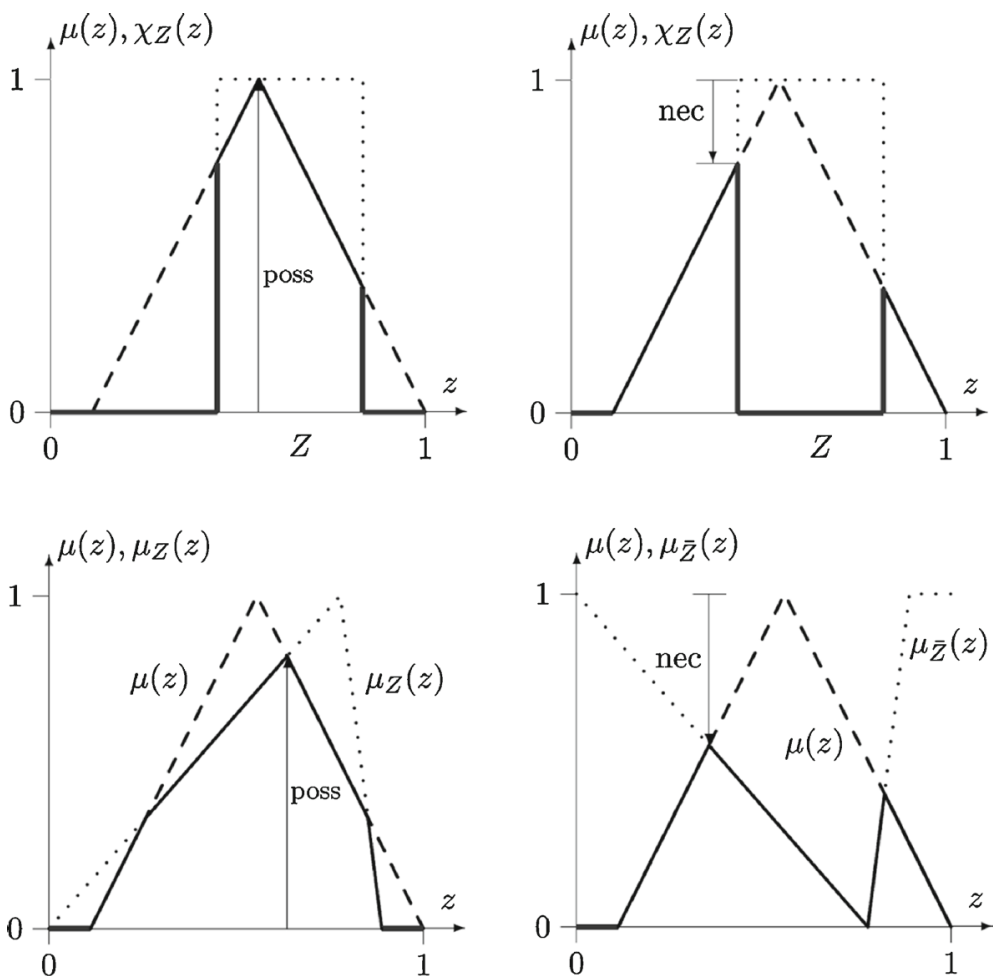

Fig. 3 Illustration for a crisp set Z: a possibility; b necessity measures; and for a fuzzy set Z: c possibility; d necessity measures

Note that when $Z=[r, 1]$, then the above index can be interpreted as a measure that element $x$ is not smaller than $r$, i.e. $r \leq x$.

Comparing these notions to the probabilistic ones, the possibility distribution corresponds to the probabilistic distribution, and the possibility measure $\operatorname{Poss}(Z)$ corresponds to the probability of the subset $Z$.

However, in the possibility theory an additional notion is introduced. Called the necessity measure, it is defined as

$$
\operatorname{Nec}(Z)=1-\operatorname{Poss}(\bar{Z})
$$

where $\bar{Z}$ is the complementary set of $Z$ in $[0,1]$, see Fig. 3b. It can be interpreted as the degree that an element is necessarily located in set $Z$. Similarly as in the possibility case, an equivalent definition may be

$$
\operatorname{Nec}(Z)=1-\sup _{z \in[0,1]} \min \left\{\mu(z), \chi_{\bar{Z}}\right\}=\inf _{z \in[0,1]} \max \left\{1-\mu(z), \chi_{Z}(z)\right\} .
$$

It can be observed in Fig. 3a and $\mathrm{b}$ that a simple property holds

$$
\operatorname{Nec}(Z) \leq \operatorname{Poss}(Z)
$$


which may be interpreted that the measures give the lower and upper bounds on uncertainty connected with the localization of an element in set $Z$. The lower one, (necessity), is the degree in the range $[0,1]$ of our conviction that the point is in set $Z$. The higher one, (possibility), is the degree of our supposition.

Now, taking a fuzzy set $Z$ instead of a crisp one, the characteristic function $\chi_{Z}(z)$ is replaced by the membership function $\mu_{Z}(z)$, providing the following definitions

$$
\begin{gathered}
\operatorname{Poss}(Z)=\sup _{z \in[0,1]} \min \left\{\mu(z), \mu_{Z}(z)\right\} \\
\operatorname{Nec}(Z)=1-\sup _{z \in[0,1]} \min \left\{\mu(z), \mu_{Z}\right\}=\inf _{z \in[0,1]} \max \left\{1-\mu(z), \mu_{Z}(z)\right\}
\end{gathered}
$$

see Fig. $3 \mathrm{c}$ and d. For further use, $\mu_{\bar{Z}(z)}=1-\mu_{Z}(z)$ is introduced as the membership function of the complementary set of $Z$.

\subsubsection{Possibility of dominance indices}

Having introduced the above notions, we can pass to a definition of fuzzy dominance indices. To calculate the possibility and necessity indices, the membership functions are analyzed on a two-dimensional plane $(z, y)$, and more specifically, either on the upper right or the bottom left half of the square $[0,1] \times[0,1]$, compare with Fig. 4a. This is analogous to consideration of two-dimensional probability density function for independent variables. To compare two fuzzy numbers, one of them, say $B$, is treated as a reference. Its membership function plays a role of a reference possibility distribution.

a

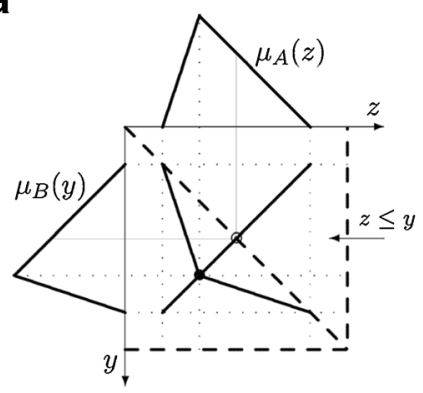

C

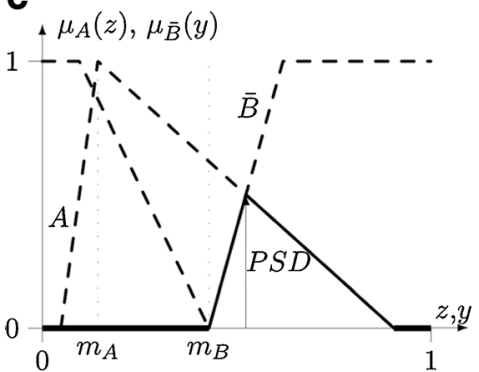

b

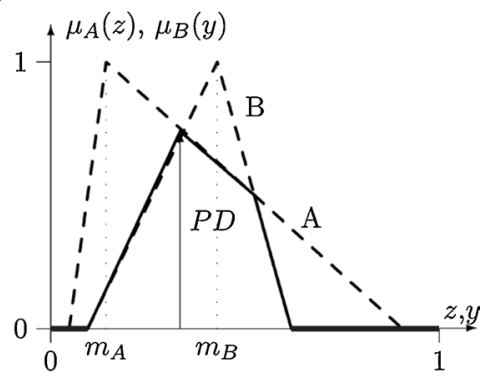

d

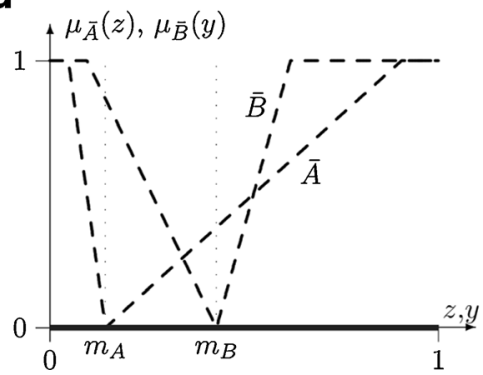

Fig. 4 Calculation of: a the $P D$ index on the $(z, y)$ plane; $\mathbf{b}$ the $P D$ index on a line; $\mathbf{c}$ the $P S D$ index; $\mathbf{d}$ the $N S D$ index 
Now we introduce the notion of the dominance of a fuzzy set $A$ over $B$, denoted below as $A \succcurlyeq B$, and the strict dominance, denoted as $A \succ B$.

The possibility of dominance $(P D)$ index of a fuzzy set $A$ over a fuzzy set $B$ is defined as

$$
P D=\operatorname{Poss}(A \geqslant B)=\sup _{z, y ; z \geq y} \min \left\{\mu_{A}(z), \mu_{B}(y)\right\} .
$$

The index $P D$ is a measure of possibility that the fuzzy numbers $A$ is greater than $B$, or that the set $A$ dominates the set $B$. This index was first proposed by Baas and Kwakernaak (1977). A probabilistic analogue of this index would be the probability that $A \geq B$. This index has to be analysed on the plane $(z, y)$ in the upper right half of the square $[0,1] \times[0,1]$, see Fig. $4 a$, where the projection of the function $\min \left\{\mu_{A}(z), \mu_{B}(y)\right\}$ on the square is drawn with the membership functions $\mu_{A}(z)$ and $\mu_{B}(y)$ drawn on the axes. The highest value of this function (equal to 1 ) is located in the area $y>z$ (at the point marked with $\bullet$ ), while the value $P D<1$ is located on the boundary of the upper half of the square, at the point marked with $\circ$. It is now easy to notice that the value $P D$ can be calculated as presented in Fig. 4b.

Analysing the way the value $P D$ is calculated, with notation from Fig. $4 \mathrm{~b}$, it is seen that

$$
\begin{aligned}
& \text { Poss }(A \geqslant B)=1 \text { if } m_{A} \geq m_{B} \\
& \text { Poss }(A \geqslant B)=0 \text { if } m_{A}+p_{r A} \leq m_{B}+p_{l B}
\end{aligned}
$$

where $p_{l B}$ is the left end of the support of $B$, and $p_{l A}$ the right end of the support of $A$, see Figure S2 in the supplementary material for illustration of $p_{l}$ and $p_{r}$. The possibility of dominance $(P D)$ equals 0 , if any point of the support of $A$ is smaller than any point of the support of $B$. When the supports overlap, $P D>0$. If the core of $A$ is greater or equal to the core of $B$, then $P D=1$.

The possibility of strict dominance (PSD) index for a fuzzy set $A$ over a fuzzy set $B$ is defined as

$$
P S D=P o s s(A \succ B)=\sup _{z} \inf _{y, y \geq z} \min \left\{\mu_{A}(z), 1-\mu_{B}(y)\right\}
$$

where $\mu_{A}(z)$ and $\mu_{B}(y)$ are the membership functions of $A$ and $B$, respectively.

Analysis of the function on the two dimensional square results in the situation depicted in Fig. 4c. Now we have

$$
\begin{array}{lll}
\text { Poss }(A \succ B)=1 & \text { if } & m_{A} \geq m_{B}+p_{r B} \\
\operatorname{Poss}(A \succ B)=0 & \text { if } & m_{A}+p_{r B} \geq m_{B}
\end{array} .
$$

where $p_{r B}$ is the right end of the support of $B$.

The possibility of strict dominance index is therefore equal to 0 , when the support of $\mathrm{A}$ is situated to the left of the core of $B$. It is positive in the opposite case. It equals 1 , if the support of $B$ is situated to the left of the core of $A$. The membership function of $A$ has to be shifted further to the right to achieve the same value of the index as in the possibility of dominance case. 


\subsubsection{Necessity of dominance indices}

The necessity of dominance (ND) index of a fuzzy set $A$ over a fuzzy set $B$ is defined as

$$
N D=\operatorname{Nec}(A \geqslant B)=\inf _{z} \sup _{y, y \leq z} \max \left\{1-\mu_{A}(z), \mu_{B}(y)\right\} .
$$

As with previous analyses, calculation of this index reduces to analysis of the situation presented in Fig. 4c. It yields

$$
\begin{aligned}
& \operatorname{Nec}(A \geqslant B)=1 \text { if } m_{A}-p_{l A} \geq m_{B}, \\
& \operatorname{Nec}(A \geqslant B)=0 \text { if } m_{A} \leq m_{B}-p_{l B} .
\end{aligned}
$$

Thus, the necessity of dominance index equals 0 when the core of $A$ is to the left of the support of $B$. It is positive in the opposite case. It equals 1 , if the support of $A$ is situated to the right of the core of $B$.

The necessity of strict dominance (NSD) index of a fuzzy set $A$ over a fuzzy set $B$ is defined as

$$
\begin{aligned}
N S D & =\operatorname{Nec}(A>B)=\inf _{z, y ; y \leq z} \max \left(1-\mu(z), 1-\mu_{B}(y)\right)= \\
& =1-\sup _{z, y ; y \leq y} \min \left\{\mu_{A}(z), \mu_{B}(y)\right\}=1-\text { Poss }(B \geqslant A) .
\end{aligned}
$$

This index is the opposite of the measure of possibility that set $B$ dominates set $A$. It was first proposed by Watson et al. (1979). The analysis of the index reduces to analysis of the situation presented in Fig. 4d. There is

$$
\begin{array}{lll}
\operatorname{Nec}(A \succ B)=1 & \text { if } & m_{A}-p_{l A} \geq m_{B}+p_{r B} \\
\operatorname{Nec}(A \succ B)=0 & \text { if } & m_{A} \leq m_{B} .
\end{array}
$$

An example, with a comparison of the above methods, can be found in the supplementary material.

\subsubsection{Checking fulfilment of a limit}

Next, the question is asked as to whether the techniques described can be used for limit verification. To this end, the limit can be interpreted as a point value, which is a fuzzy variable with a membership function

$$
\mu_{B}(z)= \begin{cases}1 & \text { if } \quad z=\tilde{L} \\ 0 & \text { if } \quad z \neq \tilde{L}\end{cases}
$$

where $\widetilde{L}$ is the rescaled value of the limit $L$. In this situation $P D=P S D$ and $N D=N S D$, so the analysis can be confined only to the necessity $N$ and possibility $P$ indices.

In Fig. 2e two cases are depicted: the limit $B_{1}$ higher than $m_{A}$, and the limit $B_{2}$ smaller than $m_{A}$. In the former case $P>0$ and $N=0$. In the latter $P=1$ and $N>0$. It becomes apparent that the necessity index is equivalent to the Adamo method with $N=1-\alpha$. The possibility index gives information on the degree of a failure to achieve the limit (recall that here the limit is achieved when $A$ is greater than $B$ ), which could be used for determining noncompliant inventories. 
Thus, we can formulate the following rules. The inventory is considered compliant if the necessity index is high enough. The inventory is considered noncompliant if the possibility index is small enough. This leads to the situation, which is fully analogous to the indecision interval in the probabilistic approach, as presented in Fig. 1e. Fixing the minimal necessity $N$ and maximal possibility $P$ indices brings us to the notion of an indecision interval, where the necessity index is too small and the possibility index is too high.

The application of the Dubois and Prade method provides useful information with respect to the compliance evaluation. Nevertheless, analysis of membership functions in three dimensions is rather cumbersome. Simple interpretations on the plane, as in Fig. 4, can help in the analysis. Necessity indices give practically the same information as in the methods of Adamo and Nahorski et al. The possibility indices can be useful for quantifying noncompliance.

\section{Conclusions}

The paper presents the methods for the comparison of uncertain emission inventories, and discusses their usefulness for evaluation of emission reduction limits. The review shows a variety of approaches and techniques. It clearly demonstrates that the comparison of the reported inventories with no account of uncertainty distributions leads to paradoxes, and it is not well scientifically grounded. Some of the approaches, like the undershooting method, have been proposed earlier (Godal et al. 2003; Nahorski et al. 2003; Jonas et al. 2010a), and adapted for emission trading, see additionally Nahorski et al. (2007); Nahorski and Horabik (2010,2011). Any use of the techniques outlined in the paper takes uncertainty into account, see Table 1, and thus inevitably necessitates changes to the presently used rules of compliance checking. To date, the verification mechanisms depend only on reported inventories. They give a decisive answer, which may, however, be difficult to support when uncertainty of the inventories is considered, as shown in Fig. 1. In terms of probability or other measures, like possibility, only weaker statements on compliance can be formulated; for example, the probability of not fulfilling the limit. This means that either conservative decisions have to be taken or indecision situations may occur. However, these lack any controversy and are thus transparent, since the inventories can be compared and ordered. Ignoring uncertainty is more hazardous for asymmetric distributions, which may occur in many national inventories. It is also of great importance for comparisons of emission uncertainty distributions representing sectors of different activities, such as energy and agriculture.

Within the fuzzy approach, some problems arise with the representation of the incomplete information on the inventories uncertainty in the form of membership functions. However, the membership functions can be constructed and interpreted as approximations to the inventory uncertainty, formulated on the basis of the best available knowledge. The present state of the development in this area allows only weak statements on comparison to be formulated, providing only some indices of possibility or necessity for instance. For decision making, one can set critical values on these indices, however, it may be more difficult than for the stochastic case due to smaller intuition on the indices interpretation.

In spite of basic conceptual differences between the probabilistic and fuzzy approaches, many techniques are surprisingly similar. Among them, the critical values and fuzzy dominance methods provide similar techniques for checking compliance, with small technical differences in terminology and decision parameters. This paper has not been intended to elaborate legislation propositions for compliance rules, due in part to restrictions on its length. Examples of analytical conditions for checking compliance can be found in the literature mentioned. 
Table 1 Comparison of methods discussed in the paper

\begin{tabular}{|c|c|c|}
\hline Group of methods & Required information & Characteristics and usefulness of the methods \\
\hline $\begin{array}{l}\text { Based on distribution } \\
\text { moments }\end{array}$ & Means and variances & $\begin{array}{l}\text { The methods use simple information but their application } \\
\text { is rather inconvenient. Two indicators can contradict } \\
\text { each other. For asymmetric distributions, mean values } \\
\text { are different from the reported (dominant) values. } \\
\text { Application of semivariance requires information on } \\
\text { the uncertainty distribution. }\end{array}$ \\
\hline Based on critical values & $\begin{array}{l}\text { Probability or possibility } \\
\text { mass of the inventory } \\
\text { uncertainty above a } \\
\text { specific value }\end{array}$ & $\begin{array}{l}\text { This group of methods seems to be particularly convenient } \\
\text { for the compliance problem; some variants of these } \\
\text { methods have been already proposed independently in } \\
\text { several papers. The methods need more advanced } \\
\text { information on the uncertainty distribution, which } \\
\text { requires more sophisticated methods for its acquisition. } \\
\text { Moreover, a good understanding of the applied inference } \\
\text { techniques is required for decision making, as the values } \\
\text { used in the compliance rules differ from the reported } \\
\text { inventories. This may be questioned on the ground of } \\
\text { deterministic common-sense arguments. }\end{array}$ \\
\hline Based on dominance & $\begin{array}{l}\text { Full distribution of } \\
\text { inventory uncertainty }\end{array}$ & $\begin{array}{l}\text { Although the same notion of dominance is used in both } \\
\text { stochastic and fuzzy approaches, the methods are very } \\
\text { different. The stochastic methods are not always decisive } \\
\text { and rather difficult for practical applications. The fuzzy } \\
\text { methods use little known notions of possibility and } \\
\text { necessity indices, and require understanding of the } \\
\text { sophisticated underlying theory. The geometrical } \\
\text { calculation of the indices proposed in the present paper } \\
\text { may make the method easier to grasp. As shown, } \\
\text { comparison of an inventory against an exact (crisp) limit } \\
\text { allows for its reduction to a variant of methods from the } \\
\text { critical values group. }\end{array}$ \\
\hline
\end{tabular}

Possible approximations of the uncertainty distributions in the fuzzy approach give rise to the question of the impact of approximations on the final compliance condition. This issue is also valid in the stochastic approach, since the required probability characteristics are not easy to be gathered by simple statistical treatment to get accurate estimates. It may be argued that this second-order uncertainty impacts the results to a lesser extent than the first-order uncertainty of the inventory itself. It seems that this question can be solved using the idea underlying the methods described in the present paper: the worse the data are, the lower the reported inventory should be to achieve a sufficient credibility. Thorough investigation of this problem is left for further studies.

Acknowledgments O. Hryniewicz, Z. Nahorski and J. Verstraete gratefully acknowledge financial support from the Polish State Scientific Research Committee within the grant N N519316735 as well as from statutory fund of the Systems Research Institute. J. Horabik was financially supported by the Foundation for Polish Science under International $\mathrm{PhD}$ Projects in Intelligent Computing, which is financed by The European Union within the Innovative Economy Operational Programme 2007-2013 and European Regional Development Fund. The authors thank the anonymous reviewers for the comments, which helped us to improve the paper.

Open Access This article is distributed under the terms of the Creative Commons Attribution License which permits any use, distribution, and reproduction in any medium, provided the original author(s) and the source are credited. 


\section{References}

Adamo JM (1980) Fuzzy decision trees. Fuzzy Sets Syst 4:207-219

Baas SM, Kwakernaak H (1977) Rating and ranking of multiple-aspect alternatives using fuzzy sets. Automatica 13:47-58

Bortolan G, Degani R (1985) A review of some methods for ranking fuzzy subsets. Fuzzy Sets Syst 15:1-19

Dubois D, Prade H (1983) Ranking fuzzy numbers in the setting of possibility theory. Inf Sci 30:183-224

Gillenwater M, Sussman F, Cohen J (2007) Practical policy applications of uncertainty analysis for national greenhouse gas inventories. Water, Air \& Soil Pollution: Focus 7(4-5):451-474

Godal O, Ermolev Y, Klaassen G, Obersteiner M (2003) Carbon trading with imperfectly observable emissions. Environ Resour Econ 25:151-169

Gusti M., Jęda W. (2002) Carbon management: new dimension of future carbon research. IR-02-006. IIASA, Austria. http://www.iiasa.ac.at/Publications/Documents/IR-02-006.pdf

Graves SB, Ringuest JL (2009) Probabilistic dominance criteria for comparing uncertain alternatives: A tutorial. Omega 37:346-257

Hryniewicz O., Nahorski Z. (2008) Verification of Kyoto Protocol - a fuzzy approach. In: L. Magdalena, M. Ojeda-Aciego, J. L. Verdegay (Eds.) Proc. IPMU'08. Torremolinos, Spain, 729-734. http://www.gimac. uma.es/ipmu08/proceedings/papers/096-HryniewiczNahorski.pdf

IPCC (1996) Revised 1996 IPCC Guidelines for national Greenhouse Gas Inventories. Vol. 1. Reporting Instructions. IPCC. Available at: http://www.ipcc-nggip.iges.or.jp/public/gl/invs4.html

Jain R (1976) Decision-making in the presence of fuzzy variables. IEEE Trans Systems Man Cybernet 6:698703

Jonas M, Nilsson S, Obersteiner M, Gluck M, Ermoliev YM (1999) Verification times underlying the Kyoto protocol: Global benchmark calculations. IR-99-062. IIASA, Austria. http://www.iiasa.ac.at/Publications/ Documents/IR-99-062.pdf

Jonas M, Nilsson S (2007) Prior to economic treatment of emissions and their uncertainties under the Kyoto Protocol: Scientific uncertainties that must be kept in mind. Water, Air, and Soil Pollution: Focus 7:495-511

Jonas M, Gusti M, Jęda W, Nahorski Z, Nilsson S (2010a) Comparison of preparatory signal analysis techniques for consideration in the (post-)Kyoto policy process. Clim Chang 103(1-2):175-213

Jonas M, Marland G, Winiwarter W, White T, Nahorski Z, Bun R, Nilsson S (2010b) Benefits of dealing with uncertainty in greenhouse gas inventories: introduction. Clim Chang 103(1-2):3-18

Lieberman D, Jonas M, Winiwarter W, Nahorski Z, Nilsson S (eds) (2007) Accounting for climate change: Uncertainty in greenhouse gas inventories - verification, compliance, and trading. Springer, Dordrecht

Nahorski Z, Horabik J (2010) Compliance and emission trading: rules for asymmetric emission uncertainty estimates. Clim Chang 103(1-2):303-325

Nahorski Z, Horabik J (2011) A market for pollution emission permits with low accuracy of emission estimates. In: Kaleta M, Traczyk T (eds) Modeling multi-commodity trade: Information exchange methods. Springer, Verlag, pp 151-165

Nahorski Z, Horabik J, Jonas M (2007) Compliance and emission trading under the Kyoto Protocol: Rules for uncertain inventories. Water, Air \& Soil Pollution: Focus 7(4-5):539-558

Nahorski Z, Jęda W, Jonas M (2003) Coping with uncertainty in verification of the Kyoto obligations. In: Studziński J., Drelichowski L., Hryniewicz O. (Eds.) Zastosowania informatyki i analizy systemowej w zarządzaniu. SRI PASm, 305-317.

Ramirez RA, de Keizer C, van der Sluijs JP (2006) Monte Carlo analysis of uncertainties in the Netherlands greenhouse gas emission inventory for 1990-2004. Copernicus Institute for Sustainable Development and Innovation, Utrecht, the Netherlands. Available at: http://www.chem.uu.nl/nws/www/publica/ publicaties2006/E2006-58.pdf

Roy AD (1952) Safety first and the holding of assets. Econometrica 20:431-449

Tran L, Duckstein L (2002) Comparison of fuzzy numbers using a fuzzy distance measure. Fuzzy Sets Syst 130: $331-341$

Watson SR, Weiss JJ, Donell ML (1979) Fuzzy decision analysis. IEEE Trans Systems Man Cybernet 9:1-9

White T, Jonas M, Nahorski Z, Nilsson S (eds) (2011) Greenhouse gas inventories. Dealing with uncertainty. Springer, Dordrecht

Winiwarter W, Rypdal K (2001) Assessing the uncertainty associated with national greenhouse gas emission inventories: A case study for Austria. Atmos Environ 35:5425-5440

Yager RR (1981) A procedure for ordering fuzzy subsets of the unit interval. Inform Sci 24:143-161 